\title{
Simultaneous optimization of structural shape and control system of large-scale space frame
}

\author{
M. Dan \& M. Kohiyama \\ Graduate School of Science and Technology, Keio University, Japan
}

\begin{abstract}
In general, large-scale space frames, such as stadiums and gymnasiums, have a role as an evacuation facility. However, some accidental falls of a ceiling and equipment during an earthquake have been reported, and these accidents might cause human injury or casualty. On the other hand, these structures often have an aesthetically elaborated design, but they do not always have high performances from a structural point of view. This research proposes a method to support designing a large-scale space frame, which implements: 1) a design elaborated by an architect and 2) structural and control performances, which can reduce damage or fall of a ceiling, on the basis of simultaneous optimization of structural and control systems. The number of design parameters in the optimization problem is reduced by describing the roof shape with a Bézier surface. The genetic algorithm is used for the optimization.

Keywords: structural engineering, earthquake engineering, architectural design, space frame, structural shape, Bézier surface, optimization, seismic control, tuned mass damper, genetic algorithm.
\end{abstract}

\section{Introduction}

Many large-scale space frames, such as stadiums and gymnasiums, have aesthetics implicated by architectural designers. At the same time, they have a role as disaster refuge facilities and they would be used as evacuation centers at the occurrence of disaster, such as an earthquake. However, damages and accidental falls of the ceiling and lighting equipment hanging on the roof structure have been reported in many earthquakes, such as the 1995 Kobe Earthquakes, the 2011 Great East Japan Earthquake and so on (Kawaguchi et al. [1]). 
To prevent these kinds of damage, several studies on seismic control of a large scale-space frame have been conducted. For example, Kusunoki et al. [2] devised a methodology of seismic control of a dorm structure using a tuned mass damper (TMD). Yoshinaka and Kawaguchi [3] proposed a seismic control system using multiple TMDs. However, these studies focused on a symmetric structure model and it has not been confirmed whether a TMD is applicable to large-scale structures with various shapes.

In addition, several studies which tried to design space frame using optimization have been conducted. For example, Osaki et al. [4] proposed a design method for a double-layer space truss. Lagaros and Papadopoulos [5] derived an optimal design of shell structures with random geometric, material and thickness imperfections.

Thus, this paper proposes a design method of a roof structure of a large-scale space frame for optimizing the structural shape and the control system of the structure simultaneously using a genetic algorithm (GA).

\section{Structural modelling}

\subsection{Bézier surface}

In this paper, a space structure is modelled with finite elements. In general, a space structure has a large number of nodes and it is not efficient to conduct optimization using all node coordinates as design variables directly. Hence, a two-dimensional Bézier surface is utilized to reduce the number of design variables in optimization. The control points of a Bézier surface are used as design variables and the coordinate of each node is calculated based on the equation of the Bézier surface. The initial shape of the structure model is shown in Figure 1. An asymmetric roof structure is adopted as an initial design and the related shape constraints is introduced in the optimization.

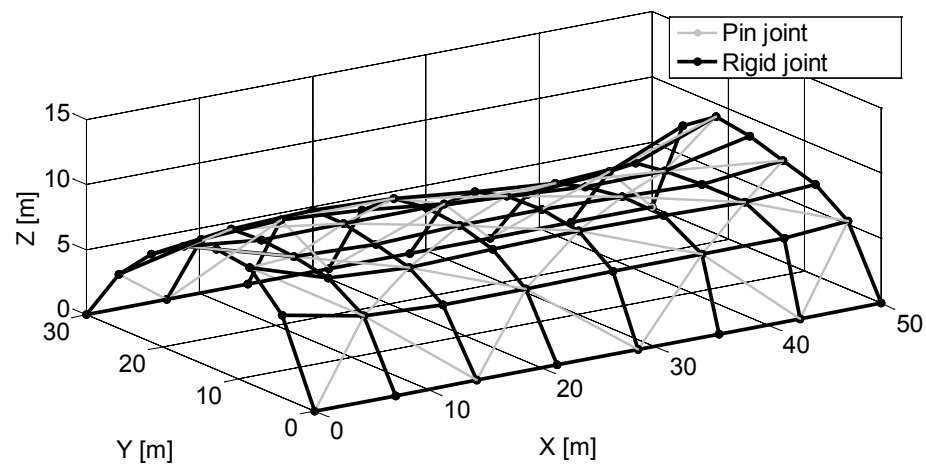

Figure 1: $\quad$ Initial shape.

A given Bézier surface of order $(n, m)$ is defined by a set of $(n+1)(m+1)$ control points $\mathbf{k}_{i, j}$. A two-dimensional Bézier surface can be defined as a 
parametric surface, on which the position vector of a point $\mathbf{p}$ is given as a function of the parametric coordinates $u$ and $v$, as follows:

$$
\mathbf{p}(u, v)=\sum_{i=0}^{n} \sum_{j=0}^{m} B_{i}^{n}(u) B_{j}^{m}(v) \mathbf{k}_{i, j}
$$

where

$$
B_{i}^{n}(u)=\left(\begin{array}{c}
n \\
i
\end{array}\right) u^{i}(1-u)^{n-i}
$$

and

$$
\left(\begin{array}{l}
n \\
i
\end{array}\right)=\frac{n !}{i !(n-i) !}
$$

are a Bernstein polynomial and a binomial coefficient, respectively.

In this paper, both $n$ and $m$ are set to 4 so that the described Bézier surface has the order $(4,4)$. As seen in the initial shape of the structural model shown in Figure 1, the model has 8 continuous beams in both $\mathrm{X}$ and $\mathrm{Y}$ directions, and there are 64 joint nodes in total. Using a Bézier surface of order $(4,4)$, the total number of the control points is 25 . Thus, the number of the design variables is substantially reduced to less than half.

\subsection{Structural parameters}

Steel tubes are assumed as the structural members. The continuous beams are rigidly connected. In addition to the continuous beams, diagonal beams are inserted with pin joints as shown in Figure 1. The total number of members is 161. Each beam member is modelled with a single element of a linear-elastic Euler beam with distributed mass. The structural parameters are shown in Table 1, and the Young's modulus and the Poisson's ratio are set to $206 \mathrm{GPa}$ and 0.3, respectively.

The Rayleigh damping is used for the structural damping, and both damping factors of the first and second modes are set to 0.02 . In this study, only roof structure is considered, and it is assumed that all the endpoint joint nodes in the $\mathrm{Y}$ direction are supported by the rigid substructure with pin joints. Regarding dead load, only self weight is considered.

Table 1: $\quad$ Structural parameters.

\begin{tabular}{lll}
\hline Parameter & & Value \\
\hline External diameter of the steel tube & $d_{1}$ & $300 \mathrm{~mm}$ \\
Thickness of the circular tube & $t_{\mathrm{s}}$ & $15 \mathrm{~mm}$ \\
Mass density of the steel material & $\rho$ & $7.85 \times 10^{3} \mathrm{~kg} / \mathrm{m}^{2}$ \\
Span of the long side of the model & $X$ & $50 \mathrm{~m}$ \\
Span of the short side of the model & $Y$ & $30 \mathrm{~m}$ \\
Height of the design space & $Z$ & $15 \mathrm{~m}$ \\
\hline
\end{tabular}




\section{Controller modelling}

\subsection{Control device}

There are numerous seismic control systems for buildings and they can be classified into active, semi-active and passive control systems depending on the presence or absence of the power input. In general, semi-active and active control systems cost higher than passive one because they need electrical power and require sensors and computers, which has to be repaired or replaced in a certain years. Hence, from the financial point of view, this paper focuses on the passive control system. In some previous research, such as in $[1,2]$, the effect of TMD on the seismic control has been confirmed for a symmetric space frame. Thus, the same type of TMD is considered in this paper.

\subsection{Design of tuned mass damper}

In general, the design of a TMD is started from deciding the mass ratio $\mu$ of a counter weight of the TMD to the whole structure including the TMD as well as objective controlled modes. To derive the optimal parameters of TMD, such as mass, damping and stiffness, a method using the fixed point theory proposed by Den Hartog [6] and some related methods based on the fixed point theory, e.g. Kida et al. [7], are well known. However, it is not easy to apply these methods directly to an asymmetric structure using multiple TMDs.

Thus, this paper proposes a design optimization method of a passive control system using multiple TMDs using GA. The mass, damping and stiffness of TMDs are included in the design variables in addition to the control points of a Bézier surface, and their values are derived directly through the optimization.

As an example, a seismic control system using two TMDs is considered in this paper. The objective controlled mode of each TMD is selected based on the largest participation factor. The TMDs are set up on the joint nodes that have the maximum amplitude in the objective mode vector, and the TMDs are assumed to move only in the vertical direction.

\section{Optimization problem setting}

\subsection{Objective function}

In this paper, an optimization problem is constructed for minimizing the maximum absolute acceleration response of the roof structure of a large-scale space frame. It has been reported that it is proper to design a TMD system based on the response to sine wave because the analysis result does not depend on the properties of the external force (Kusunoki et al. [2]). Thus, the response to sine wave input is adopted as an objective function and the optimization aims to minimize the response value. 


\subsection{Design variables}

This paper considers two different optimization problems. One is a case of a system without any TMD; this is a single objective optimization of the structural shape. The other is a case of a system with TMDs; this is a simultaneous optimization of the structural shape and control system.

In both cases, the control point coordinates of a Bézier surface are selected as design variables. In addition, the mass, damping and stiffness of the TMDs are included in the design variables only in the second case, a system with TMDs.

\subsection{Constraints}

One of the aims of this paper is to provide a useful tool to satisfy the design intention of an architectural designer with respect to a structural shape. This is realized through the optimization process by setting constraints for the variation between the positions of each node of the initial and optimal shapes. In this paper, the maximum variation is set to one-fourth of the span of the structure.

\subsection{Optimization problem}

The optimization problem setting is shown in Table 2 .

Table 2: $\quad$ Optimization problem setting.

\begin{tabular}{ccc}
\hline & System without TMD & System with TMDs \\
\hline $\begin{array}{c}\text { Objective } \\
\text { function }\end{array}$ & $J=\max _{t, i}\left\{\ddot{u}_{i}(t)+\ddot{u}_{\mathrm{g}}(t)\right\}$ & $J=\max _{t, i}\left\{\ddot{u}_{i}(t)+\ddot{u}_{\mathrm{g}}(t)\right\}$ \\
Constraints & $\left\|\boldsymbol{x}-\boldsymbol{x}_{0}\right\|<\min \left(\frac{X}{4}, \frac{Y}{4}, \frac{Z}{4}\right)$ & $\left\|\boldsymbol{x}-\boldsymbol{x}_{0}\right\|<\min \left(\frac{X}{4}, \frac{Y}{4}, \frac{Z}{4}\right)$ \\
Design & $\boldsymbol{B}_{0,0}, \boldsymbol{B}_{0,1}, \ldots, \boldsymbol{B}_{4,4}$ & $\boldsymbol{B}_{0,0}, \boldsymbol{B}_{0,1}, \ldots, \boldsymbol{B}_{4,4}$ \\
variables & $\boldsymbol{m}_{\mathrm{TMD}}, \boldsymbol{c}_{\mathrm{TMD}}, \boldsymbol{k}_{\mathrm{TMD}}$ \\
\hline
\end{tabular}

where $u_{i}(t), u_{\mathrm{g}}(t), \boldsymbol{x}$ and $\boldsymbol{x}_{0}$ are a component of the nodal displacement vector, the ground displacement, the nodal position vectors of the optimal and initial shapes, respectively. $\boldsymbol{B}_{0,0}, \boldsymbol{B}_{0,1}, \ldots, \boldsymbol{B}_{4,4}$ are the control point vectors of a Bézier surface of order $(4,4) . \boldsymbol{m}_{\mathrm{TMD}}, \boldsymbol{c}_{\mathrm{TMD}}$ and $\boldsymbol{k}_{\mathrm{TMD}}$ are the mass, damping and stiffness vector of TMDs, respectively.

\section{Optimization results}

\subsection{Results of the optimization}

Figure 2 shows the initial shape again and an asterisk $(*)$ shows the node that has the maximum response in the time history response analysis under seismic 
ground motion explained in the next section. In this case, the maximum response occurred in the up-down (the $\mathrm{Z}$ direction) component.

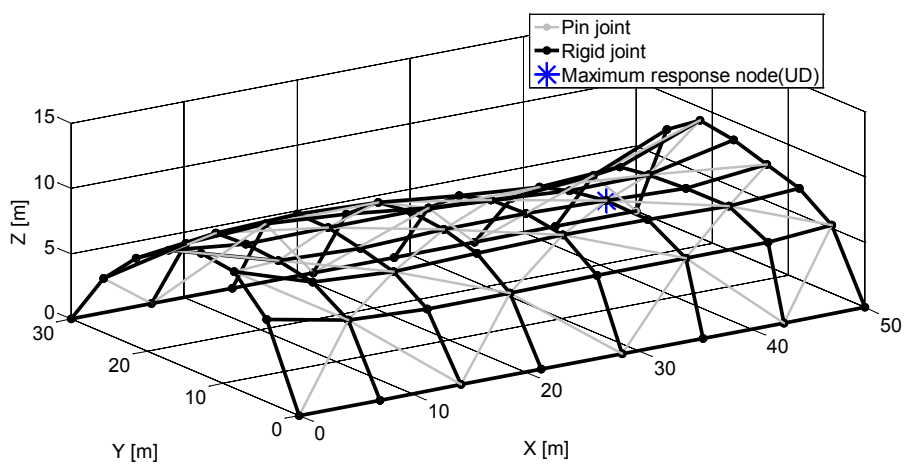

Figure 2: $\quad$ Initial shape and the maximum response node.

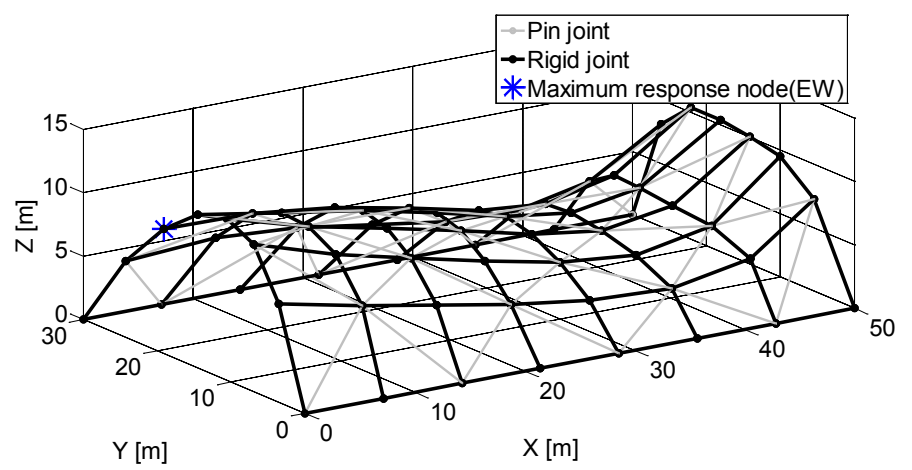

Figure 3: Optimal shape in the case of a system without TMD.

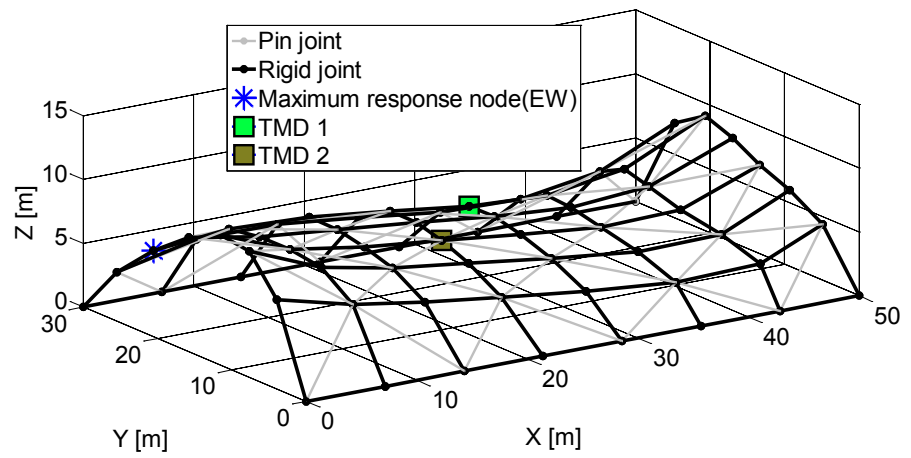

Figure 4: Optimal shape in the case of a system with TMDs. 
The optimal shapes without TMD and with TMDs are shown in Figures 3 and 4 , respectively. In these cases, the maximum response occurred in the east-west direction (the Y direction) component.

The optimal shape with TMDs is more similar to the initial shape than without TMD. Thus, TMD enables to keep a structural shape that implements design demand of an architectural designer when used as a seismic control system.

The optimal parameters of TMDs derived by GA are shown in Table 3 . These can be derived directly through a GA optimization method.

Table 3: $\quad$ Optimal parameters of TMDs.

\begin{tabular}{lcc}
\hline & TMD 1 & TMD 2 \\
\hline Controlled mode & 1st & 2nd \\
Mass [kg] & 18.74 & 17.00 \\
Damping [Ns/m] & 4.84 & 8.76 \\
Stiffness [N/m] & 80.30 & 225.79 \\
\hline
\end{tabular}

\subsection{Response under seismic ground motion}

To confirm the seismic performance of the structural shape and the effectiveness of the control system of TMDs, a time history analysis is conducted. The El Centro 1940 record is selected as input wave. The input wave is normalized so that the maximum velocity of each component becomes $0.25 \mathrm{~m} / \mathrm{s}$. The NS, EW and UD component waves are input in the directions of $\mathrm{X}, \mathrm{Y}$ and $\mathrm{Z}$, respectively.

The results of the time history response analysis are shown in Figures 5 and 6. In Figure 6, the time span is zoomed in which the peak response value is included. It is confirmed that the optimization method proposed in this paper is effective for reducing the seismic response. Especially, the peak response values of both optimal shapes without TMD and with TMDs are significantly reduced from that of the initial shape. However, in the time 15 to $22 \mathrm{~s}$, the response of the optimal shape with TMDs is larger than that of the optimal shape without TMD. This is possible because the frequency characteristic of the input ground motion would affect to the response; further research should be conducted using various input ground motions.

In addition, the maximum response value of the optimal shape with TMDs is smaller than that of the optimal shape without TMD. Thus, the effectiveness of TMD is also confirmed and it can be concluded that the optimization method proposed in this paper is useful for the design of an asymmetric large-scale space frame.

The maximum response values in the time history analysis are shown in Table 4. Although the optimal shape with TMDs has the smallest value among the three cases with respect to the maximum absolute acceleration, the maximum absolute strain value is worse than that of the initial shape. This is likely due to the low correlation between the acceleration response of a node, which is adopted as an objective function, and strain of a beam. For practical use, an improved objective function and constraints should be considered. 


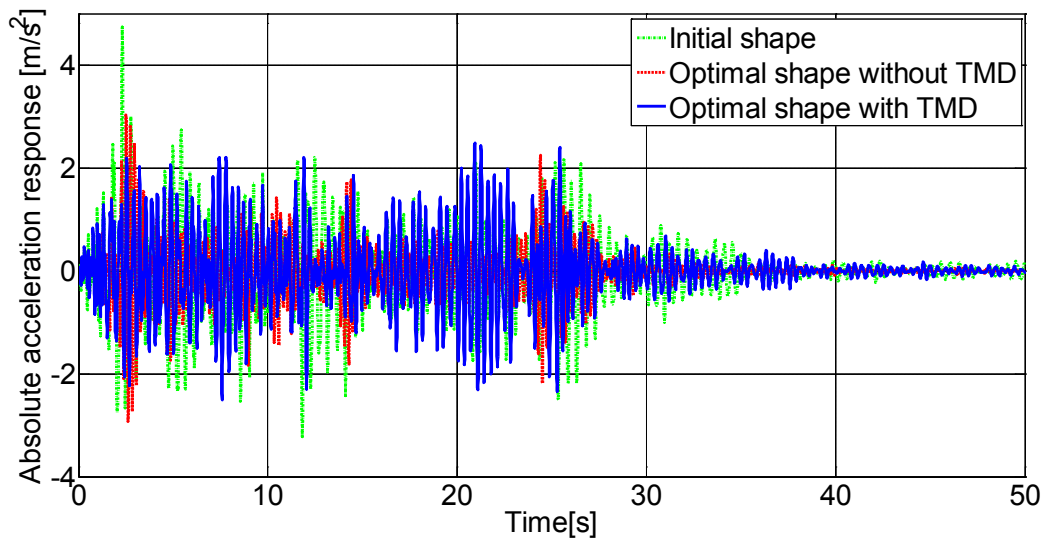

Figure 5: Results of time history response analysis.

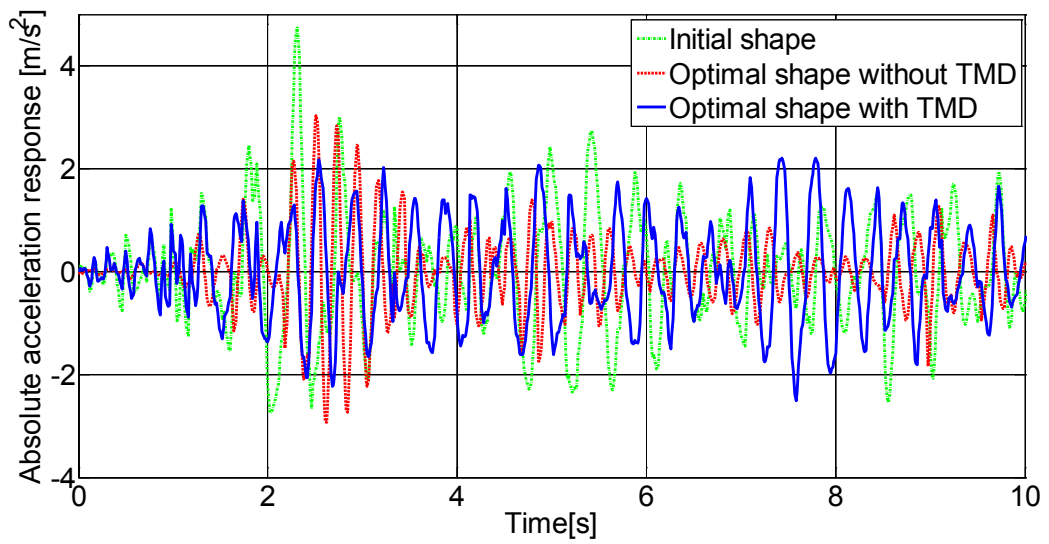

Figure 6: Results of time history response analysis; the time span including the peak response value is zoomed.

Table 4: $\quad$ Maximum response values.

\begin{tabular}{cccc}
\hline Maximum response & $\begin{array}{c}\text { Initial } \\
\text { shape }\end{array}$ & $\begin{array}{c}\text { Optimal shape } \\
\text { without TMD }\end{array}$ & $\begin{array}{c}\text { Optimal shape } \\
\text { with TMDs }\end{array}$ \\
\hline $\begin{array}{c}\text { Absolute acceleration of node } \\
{\left[\mathrm{m} / \mathrm{s}^{2}\right]}\end{array}$ & 4.74 & 3.04 & 2.51 \\
$\begin{array}{c}\text { Strain of beam } \\
{\left[10^{-6}\right]}\end{array}$ & 88.73 & 123.43 & 100.03 \\
\hline
\end{tabular}




\section{Conclusions}

In this paper, a design method of asymmetric large-scale space frames is proposed. The method is based on the optimization using GA and the structural shape and a control system using TMDs are optimized simultaneously. The maximum response to sine wave is adopted as an objective function. With respect to design variables, control node coordinates of a Bézier surface and parameters of TMDs are considered; a Bézier surface can reduce the total number of design variables and implement a similar shape to the initial design provided by an architect with optimization constraints.

In a verification study, a space frame structure with 64 joint nodes and 161 beams are examined. It was confirmed that the proposed method could provide the optimal shape that minimized the maximum absolute acceleration response to sine wave input. In addition, time history analysis was conducted using seismic ground motion as input wave. As a result, it was also confirmed that the maximum absolute acceleration response to the seismic ground motion was reduced in the optimal shapes, and the presence of TMDs enabled to reduce the peak response value and to keep similarity to the initial shape.

In future research, it is needed to consider other control systems, such as semi-active and active control systems, and to compare these control systems based on their life-cycle-cost. In addition, it is important to set clear constraints which can help architectural designers to design more aesthetic and highperformance structures. Furthermore, to introduce a semi-active or active control system to the large-scale space frame, the optimization of a whole structure of both roof and substructure should be considered.

\section{Acknowledgement}

This work is supported in part by a Grant-in-Aid for the Leading Graduate School program for "Science for Development of Super Mature Society" from the Ministry of Education, Culture Sport, Science and Technology in Japan.

\section{References}

[1] Kawaguchi, K., Taniguchi, Y., Ozawa, Y., Nakaso, Y. and Watanabe, S., Failure of Suspended Ceilings in Large Public Spaces by Great East Japan Earthquake. Seisan Kenkyu, 63(6), pp. 765-772, 2011 (in Japanese).

[2] Kusunoki, T., Xue, S. and Yamada, M., Study of Seismic Response and Vibration Control of Single-Layer Latticed Domes using TMD. Journal of Structural Engineering, 41B, pp. 17-22, 1995 (in Japanese).

[3] Yoshinaka, S. and Kawaguchi, K., Vibration Control of Spatial Structures using Spatially Distributed MTMDs. Memoirs of the Faculty of Engineering Osaka City University 49, pp. 19-28, 2008.

[4] Ohsaki, M., Nakamura T., and Kohiyama, M., Shape Optimization of a Double-Layer Space Truss Described by a Parametric Surface, International Journal of Space Structures, 12(2), pp. 109-119, 1997. 
178 Earthquake Resistant Engineering Structures IX

[5] Lagaros, N. D. and Papadopoulos, V., Optimum design of shell structures with random geometric, material and thickness imperfections, International Journal of Solids and Structures, 43, pp. 6948-6964, 2006.

[6] Den Hartog, J. P., Mechanical Vibration, 4th edition, McGraw-Hill: New York, 1956.

[7] Kida, H., Nakaminami, S., Saito, K., Ikago, Y. and Inoue, N., A Seismic Control System with Multi-Tuning Viscous Mass Damper and Its Design Method. Journal of Structural and Construction Engineering, Transaction of Architectural Institute of Japan, (643), pp. 1575-1583, 2009 (in Japanese). 